\title{
Acute Ischemic Stroke Associated with COVID-19
}

\author{
Buse Cagla Ari \\ Department of Neurology, Medical Faculty, Bahcesehir University, Pendik Medical Park Hospital, Istanbul, Turkey
}

\begin{abstract}
During the prevailing coronavirus disease 2019 (COVID-19) pandemic, the disease has started manifesting with some neurological symptoms. There have been reports on acute ischemic stroke, cerebral venous thrombosis, and intracerebral hemorrhage associated with COVID-19. The plausible mechanism that causes these ischemic processes is called "sepsis-induced coagulopathy." A 40-year male patient, who was hospitalised due to COVID-19 pneumonia, developed sudden-onset motor aphasia and right-sided hemiplegia. He was then placed in, with the diagnosis of acute ischemia, most probably associated with COVID-19, considering that the patient's medical history was not remarkable for a relevant etiology, and all tests for the etiology of ischemic stroke showed normal findings. The patient was placed on therapy with acetyl salicylic acid, $300 \mathrm{mg} / \mathrm{day}$. It is presumed that ischemic events occur by an increase in coagulopathy secondary to inflammation. COVID-19 causes ischemic processes by inducing endothelial dysfunction and arterial or venous thrombosis.
\end{abstract}

Key Words: COVID-19, Stroke, Coagulopathy, Ischemia; SARS-CoV-2.

How to cite this article: Ari BC. Acute Ischemic Stroke Associated with COVID-19. J Coll Physicians Surg Pak 2021; 31(JCPSPCR):CR132-CR134.

\section{INTRODUCTION}

Coronavirus disease 2019 (COVID-19) caused by the severe acute respiratory syndrome coronavirus 2 (SARS-CoV-2) presents with respiratory problems, but it can also manifest with neurological symptoms as anosmia, seizures, and encephalopathy. There have been reports of cases with ischemic stroke, cerebral venous thrombosis, and intracerebral hemorrhage., There is an increase in cerebrovascular events related to the disease. ${ }^{3}$ The underlying pathophysiology has been defined as "sepsis-induced coagulopathy(SIC)," which has been known as the initial process of disseminated intravascular coagulation (DIC). The systemic inflammatory response induced by infection aggravates coagulopathy, and results in organ failure, and ischemia by causing endothelial dysfunction. ${ }^{3,4}$ So far, a few cases of acute ischemic stroke associated with COVID-19 have been reported, but the actual underlying mechanism has not been clarified yet. The current report aims to increase the awareness of the neurological manifestations of COVID-19.

\section{CASE REPORT}

A 40-year male patient presented with fever, shortness of breath, and cough that started a week before admission. On examination, he had dyspnea (respiratory rate, 28/min), fever $\left(37.6^{\circ} \mathrm{C}\right.$ ), and hypoxemia (oxygen saturation on room air $=80 \%$ ).

Correspondence to: Dr. Buse Cagla Ari, Department of Neurology, Medical Faculty, Bahcesehir University, Pendik Medical Park Hospital, Istanbul, Turkey

E-mail: juvelia@gmail.com

Received: December 22, 2020; Revised: March 02, 2021; Accepted: April 18, 2021

DOI: https://doi.org/10.29271/jcpsp.2021.JCPSPCR.CR132
In the laboratory, procalcitonin was $0.59 \mathrm{ng} / \mathrm{ml}$, C-reactive protein (CRP) was $265.1 \mathrm{mg} / \mathrm{L}$, ferritin was $1180.5 \mu \mathrm{g} / \mathrm{l}$, troponin was $19.2 \mathrm{ng} / \mathrm{l}$, D-dimer was $1280 \mu \mathrm{g} / \mathrm{dl}$, prothrombin time (PT) was 16.1 seconds, and fibrinogen was $661 \mathrm{mg} / \mathrm{dl}$. The complete blood count revealed leukocytosis $\left(9.22 \times 10^{9} \mathrm{~L}\right)$, lymphopenia $\left(1.04 \times 10^{9} / \mathrm{L}\right)$, and thrombocytopenia $\left(100 \times 10^{9} / \mathrm{L}\right)$. Thoracic computed tomography (CT) revealed bilateral consolidation in the upper, middle, and lower zones of the lungs, accompanied by air bronchograms and infiltration, mainly of lung parenchyma in subpleural areas with ground glass opacity in the middle and upper zones (Figure 1). The patient was diagnosed with COVID-19 upon detection of SARS-CoV-2 in reverse transcriptase-polymerase chain reaction (RT-PCR) analysis of the throat swab sample, and was placed on therapy with azithromycin $250 \mathrm{mg} /$ day, favipiravir $600 \mathrm{mg} /$ day followed by the loading dose. He was provided with continuous positive airway pressure ventilation support to relieve respiratory distress. During his stay in the hospital, he developed motor aphasia, forced deviation of the head and eyes to the left, right homonymous hemianopsia, right central facial paralysis, right hemiplegia, brisk deep tendon reflexes in right extremities, and extensor plantar response in the right foot on the fifth day of admission. The National Institutes of Health Stroke Scale (NIHSS) score was 16. Blood pressure was $135 / 80 \mathrm{~mm} / \mathrm{Hg}$. Electrocardiogram revealed a normal sinus rhythm. We observed an acute diffusion restriction in the area supplied by the upper division of the left middle cerebral artery (MCA) in multiple resonance imaging (MRI) (Figure 2). The patient was not treated with intravenous (iv) thrombolytic therapy as he was already on therapy with enoxaparin 12,000 IU/day. We continued the existing therapy with low-molecular-weight heparin (LMWH), and added acetyl salicylic acid $300 \mathrm{mg} /$ day with the diagnosis of 
acute ischemic stroke. Cranial and cervical CT angiography showed no findings suggestive of a vascular occlusion. His cholesterol levels and $\mathrm{HbAlc}$ were within normal ranges. He tested negative for the vasculitis workup. Transthoracic echocardiography $(\mathrm{ECHO})$ revealed normal findings. The history was not remarkable for any disease or drug use. He was considered to have acute ischemic stroke secondary to COVID-19, considering the nature of existing clinical symptoms and the lack of findings that would suggest other causes of stroke. He was connected to the mechanical ventilator upon a further decline in the consciousness level. On the $10^{\text {th }}$ day, his consciousness level improved, and muscle strength increased to $3 / 5$ in the upper and 2/5 in the lower limbs.

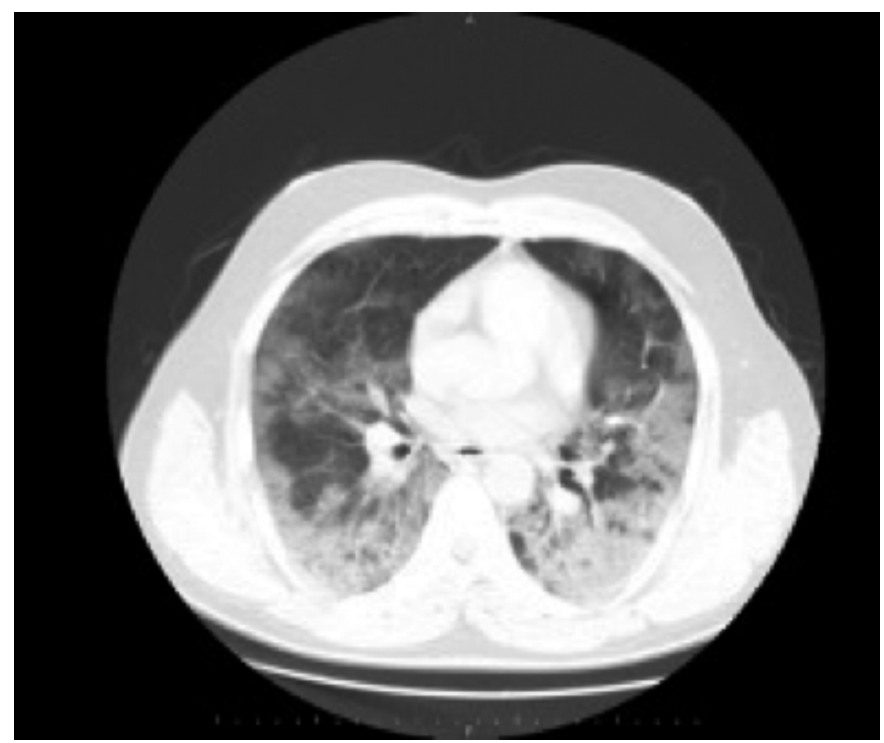

Figure 1: A single axial image from the thorax CT scan of the patient demonstrating ground-glass opacity on basal parts of the lungs bilaterally.

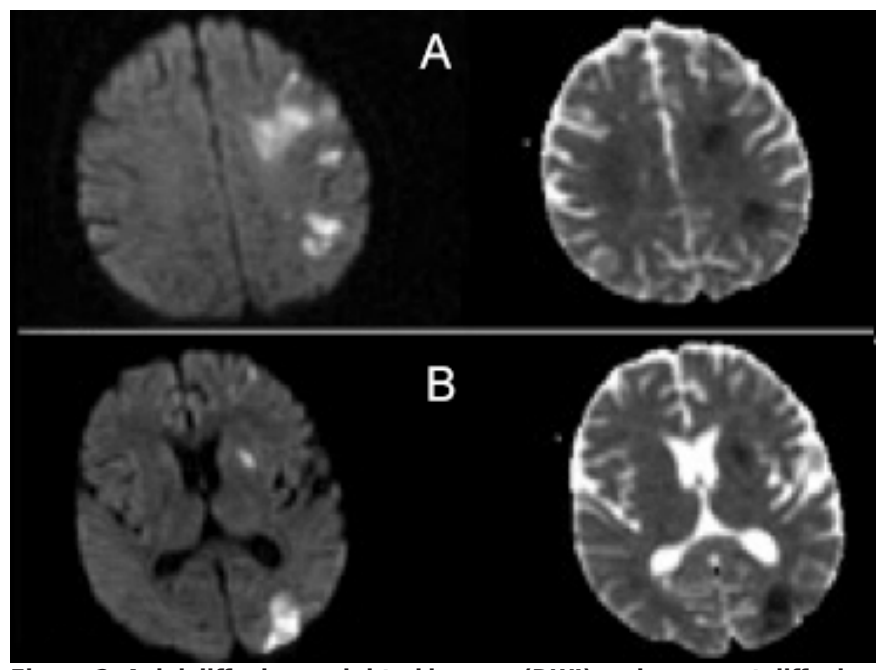

Figure 2: Axial diffusion-weighted images (DWI) and apparent diffusion coefficient (ADC) images on MRI of the patient. It demonstrates increased signal in the territory of the upper division of left middle cerebral artery (MCA) on A and B.

\section{DISCUSSION}

Recent studies have reported a mean age of 71 years in patients with COVID-19-related stroke. It has also been observed that patients with comorbid conditions exhibit a more aggressive disease course and are prone to ischemic events. ${ }^{5,6}$ Our patient did not have a risk factor, tested negative in the young stroke panel, and the tests to detect etiology identified no factor to cause coagulopathy. To the best of our knowledge, the present patient was one of the first cases of large-vessel artery ischemia secondary to COVID-19, younger than 50-year, and that did not have any risk factors. ${ }^{7,8}$

A proposed mechanism of ischemic events in COVID-19 is the release of cytokines due to the activation of an inflammatory cascade secondary to hypoxia. ${ }^{9}$ An increase in interleukin (IL) levels causes the activation of endothelial and mononuclear cells, thereby increasing thrombin production, accelerating atherosclerosis, and resulting in stroke. ${ }^{8,10}$ Our patient had refractory hypoxia, and oxygen saturation did not reach the desired level, despite supplementation. It was, therefore, thought that a hypoxic state and inflammation might cause an ischemic stroke. SIC occurs after the development of the disease that leads to DIC with an increase in D-dimer and fibrinogen levels. SIC causes abnormalities in the coagulation cascade, resulting in prolonged prothrombin time (PT), increased D-dimer levels, and thrombocytopenia. COVID-19 triggers a systemic inflammatory response that lead to endothelial dysfunction and thrombosis. ${ }^{3}$ PT was prolonged, and Ddimer and fibrinogen levels were increased in our patient. The therapy with LMWH was, therefore, continued.

An issue that struck our mind was, even though our patient was on LMWH treatment, he had a stroke. Therefore, it could be concluded that LMWH therapy might not be effective enough to protect the patients from cerebrovascular events. As a result, for future approaches, at the onset of the COVID-19, it could be a rational suggestion to add anti-aggregants apart from LMWH.

In conclusion, the cerebrovascular events' pathophysiology secondary to COVID-19 is still unknown, and further studies are required to elucidate the actual mechanism.

\section{ACKNOWLEDGEMENT:}

This case report was written at Neurology Department, Siirt Research and Training Hospital, Turkey

\section{PATIENT'S CONSENT:}

The informed consent was provided from the patient.

\section{CONFLICT OF INTEREST:}

The author declared no conflict of interest.

\section{AUTHOR'S CONTRIBUTION:}

BCA: Conceived, designed the manuscript, and did editing.

\section{REFERENCES}

1. Avula A, Nalleballe K, Narula N, Sapozhnikov S, Dandu V, Toom S, et al. COVID-19 presenting as stroke. Brain Behavior Immunity 2020; 87:115-9. doi:10.1016/j.bbi. 2020.04.077.

2. Qureshi Al, Abd-Allah F, Alsenani F, Aytac E, Borhani- 
Haghighi A, Ciccone A, et al. Management of acute ischemic stroke in patients with COVID-19 infection: Report of an international panel. Int J Stroke 2020; 15(5):540-554. doi:10.1177/1747493020923234.

3. Hess DC, Eldahshan W, Rutkowski E. COVID-19-related stroke. Trans/ Stroke Res 2020; 11(3):322-5. doi:10.1007/ s12975-020-00818-9.

4. Divani AA, Andalib S, Di Napoli M, Lattanzi S, Hussain MS, Biller J, et al. Coronavirus disease 2019 and stroke: Clinical manifestations and pathophysiological insights. J Stroke Cerebrovascular Diseases 2020; 29(8):104941. doi:10. 1016/j.jstrokecerebrovasdis.2020.104941.

5. Mao L, Jin H, Wang M, Hu Y, Chen S, He Q, et al. Neurologic manifestations of hospitalized patients with coronavirus disease 2019 in Wuhan, China. JAMA Neurol 2020; 77(6):683-90. doi:10.1001/jamaneurol.2020.1127.

6. Li Y, Li M, Wang M, Zhou Y, Chang J, Xian Y, et al. Acute cerebrovascular disease following COVID-19: a single center, retrospective, observational study. Stroke Vascular Neurol 2020; 5(3):279-84. doi: 10.1136/svn-2020-000431.

7. Oxley TJ, Mocco J, Majidi S, Kellner CP, Shoirah H, Singh P, et al. Large-vessel stroke as a presenting feature of COVID-19 in the young. N Engl J Med 2020; 382(20):e60. doi:10.1056/NEJMc2009787.

8. Beyrouti R, Adams ME, Benjamin L, Cohen H, Farmer SF, Goh YY, et al. Characteristics of ischaemic stroke associated with COVID-19. J Neurol Neurosurg Psychiatry 2020; 91(8):889-91. doi:10.1136/jnnp-2020-323586.

9. Zhai P, Ding Y, Li Y. The Impact of COVID-19 on Ischemic Stroke. Diagn Pathol 2020; 15(1):78. doi:10.21203/rs. 3.rs-20393/v1.

10. Huang C, Wang Y, Li X, Ren L, Zhao J, Hu Y, et al. Clinical features of patients infected with 2019 novel coronavirus in Wuhan, China. Lancet 2020; 395(10223):497-506. doi.org/10.1016/S0140-6736 (20)30183-5. 